\title{
Effect of Aging on Feasibility and Contribution of Joint Mechanisms in Balanced Standing Using Biomechanical Modeling
}

\author{
Mohammed N Ashtiani, ${ }^{1}$ and Mahmood Reza Azghani ${ }^{1{ }^{1}}$ \\ ${ }^{1}$ Department of Biomechanics, Faculty of Mechanical Engineering, Sahand University of Technology, Tabriz, Iran \\ "Corresponding author: Mahmood Reza Azghani, Associate Professor, Department of Biomechanics, Faculty of Mechanical Engineering, Sahand University of Technology, P. O. \\ Box 51335-1996, Tabriz, Iran. Tel: +98-4133459491, Fax: +98-4133444309, E-mail: azghani@sut.ac.ir
}

Received 2017 April 16; Revised 2017 June 18; Accepted 2017 August 23.

\begin{abstract}
Background: Reduced muscle strength due to aging may adversely affect the ability of the body in postural balance.

Objectives: The aim of the present study was to numerically investigate the effects of aging on muscle recruitment patterns in keeping the balance during static standing posture.

Methods: In this numerical study, a total of 4096 static postures were considered by changing in the angles of three lower limb joints i.e. ankle, knee and hip in two complete human musculoskeletal models of young and old body with 44 muscles in the leg. Inverse dynamics approach was used to find the muscle activation in all postures. The joint mechanisms were assessed by considering the ratio between related muscle activities to the total activation.

Results: Results showed that the muscle efforts in both models were posture-dependent and the knee played a relieving role specifically for the aged model. The possible postures that the young person could provide balance was wider than the elders. Also, the aged model expended more effort in doing the same tasks. The muscles revealed distinct joint mechanisms in the young model in contrast to the elders which used higher but non-distinguishable joint mechanisms.

Conclusions: Collaboration between the joint mechanisms was higher in the aged model but the young could more rely on individual mechanisms during static postural balance.

Keywords: Posture, Aging, Muscle
\end{abstract}

\section{Background}

The ability to keep balance may be intervened by agerelated problems [1]. Risk of fall is higher between the individuals aged at least 65 years old [2]. Vision, vestibular system in the inner ear and proprioception may be considerably deteriorated by aging [3-5] which are the main biofeedback information to control the balance in human standing. Besides, aging is often followed by muscular weakness caused by decrease in cross-sectional area [6], total mass of the muscles [7] and number of motor units $[8,9]$. Muscle weakness can enhance the risk of fall up to $440 \%$ [10]. A 22-year-old follow-up study on 936 subjects developed by Stenholm et al. (2012) showed that decrease in muscle strength is mostly related to the lifestyle, and hence, considerable percent of the population may be confronted with this problem [11].

Several studies have been devoted to determine a relationship between the aging and the weakness signs. Evans and Lexell (1995) stated that the aging atrophy was led to reduction in muscle strength which impairs older individuals' mobility and increases risk of falls [12]. The ultrasonography of the quadriceps muscle in older men and women showed $25 \%-35 \%$ reduction in the cross-sectional area compared to the young $[13,14]$. By measuring elbow and knee torque, Frontera et al. (1991) reported that muscle strength in elders is $27 \%$ lower than the adults [15]. Decrease in muscle strength, on the other hand, induced loss of postural balance and control [16-19].

Routine standing strategies that the young adults employ to keep balance may be altered by aging. Although numerous role-playing factors cause age-related changes in standing, it is a great consensus on the fact that the elders use hip strategy rather than the ankle which is more common and reliable in the young. This result has been achieved by measuring kinematic changes in the lower body joints, electromyography of the muscles and also the center of pressure (CoP) excursions [20, 21]. In normal standing under perturbation, the joint rotations and the CoP movement were considerably more in the old adults, although the co-contracted muscle activations were higher in the young [22]. These authors declared that the disability to keep balance among the aged population may be originated from the latency of the muscle activations and also the muscle strength reduction [23-25]. 


\section{Objectives}

Previous studies have developed a wide range of valuable information based on experiments that inspect fall risks or standing strategies often in a phenomenological manner or model-based recognitions [25-27]. However, a mechanistic look at the elder standing can confer a detailed quantitative understanding about the role of joint strategies in the aged models. Previous works also merely focused on ankle and hip mechanisms and excluded the effects of the knee joint that was emphasized in the young postural balance $[28,29]$. Use of a precise biomechanical model may be beneficial here to investigate the effects of a certain aging factor avoiding difficulties emerge in the experiments. Therefore, the goal of the present paper is to determine the contribution of joint mechanisms in feasible standing postures by noting on the role of knee flexion in the young and old persons using a parametric biomechanical modeling. This study was about to answer these questions:

a) What is the different between the normal and weakened muscles in the number of feasible postures in static standing?

b) Does the age-related muscular weakness affect the joint mechanisms in providing equilibrium for the static cases of standing model?

c) Which of the muscles are more vulnerable in the aged model?

\section{Methods}

\subsection{Postures}

An automated home-made code was first utilized to produce all postures existed in forward leaning of the human body within the sagittal plane. Each posture was represented by three angles of the lower joints - ankle, knee and hip. Each joint was segregated into 16 discrete 6degree angle steps from 0 to 90 degrees. Therefore, a total 4096 static postures $(16 \times 16 \times 16)$ were analyzed using the biomechanical modeling. The process of producing all postures, called pre-analysis, was entirely based on the ISB recommended joint coordinates [30].

\subsection{Biomechanical Model}

The produced kinematic data in the pre-analysis section were fed into a precise biomechanical model of human musculoskeletal system (AnyBody, version 5.3, Aalborg, Denmark). The software employed anthropometric data of 50th percentiles of the European males and contained major muscles (44 muscles in the lower limbs). Bi-articular muscles were assumed to share their efforts based on the moment arms multiplied by their angle cosines of the corresponded passing joints. The muscles were considered using Hill-type model with parallel passive elasticity of the muscle, serial elasticity of the tendon and pennation angle of the fibers. Figure $1 \mathrm{~A}$ shows a typical posture in the model. Optimized inverse dynamics approach was utilized to find muscle forces. The optimization, to master the redundancy of such models, was aimed at minimizing a polynomial cost function, $\mathrm{G}$, as the sum of muscle tensions with power two, as Formula 1

$G\left(F_{i}\right)=\sum\left(\frac{F_{i}}{F_{0, i}}\right)^{2}$

where $\mathrm{F}_{0, i}$ and $\mathrm{F}_{i}$ are maximum and recruited force of muscle $i$, by satisfying the mechanical equilibrium condition

$\mathrm{CF}_{i}=\mathrm{d}$

Which $\mathrm{C}$ denotes the force coefficients matrix formed by active parts of the muscle tension as a function of muscle length and $d$ represents the weight and passive muscle force matrix [31]. The elder models were also distinguished from the young by $35 \%$ reduction in the overall muscle strengths [13].

\subsection{Feasibility Measure}

Feasible postures were filtered from the others based on a physiological limit imposed on all muscles in the model. This physiological measure accepted maximum $95 \%$ of any muscle activation, $\alpha_{i}$, in each posture, that is Formula 2

$F_{i}<0.95 \times F_{i, \max }$ ord $_{i}<0.95$

Those postures that at least one muscle activation exceeded the defined limit were considered as unfeasible of fallen postures. The model has considered related upper force bonds for each muscle based on its physiological cross-sectional area. Furthermore, maximum 35-degree ankle dorsiflexion was assumed as the heel-off limit [32], that kinesiologically constrained the outputs.

\subsection{Geometrical Analogy}

The extracted feasible regions could then be replaced by geometrically analogous ellipses in each constant knee angle (CKA). The ellipses were defined as their centers be located on the centroids of the feasible regions. The minor and major axes were also optimized so that the relative error between the areas trapped within both curves become less than $1 \%$. In other words, the area of the ellipse is close to the area that is trapped by the feasible posture with less than $1 \%$ error. The ellipses were oriented along the longitudinal expansion of the feasible regions. 
A

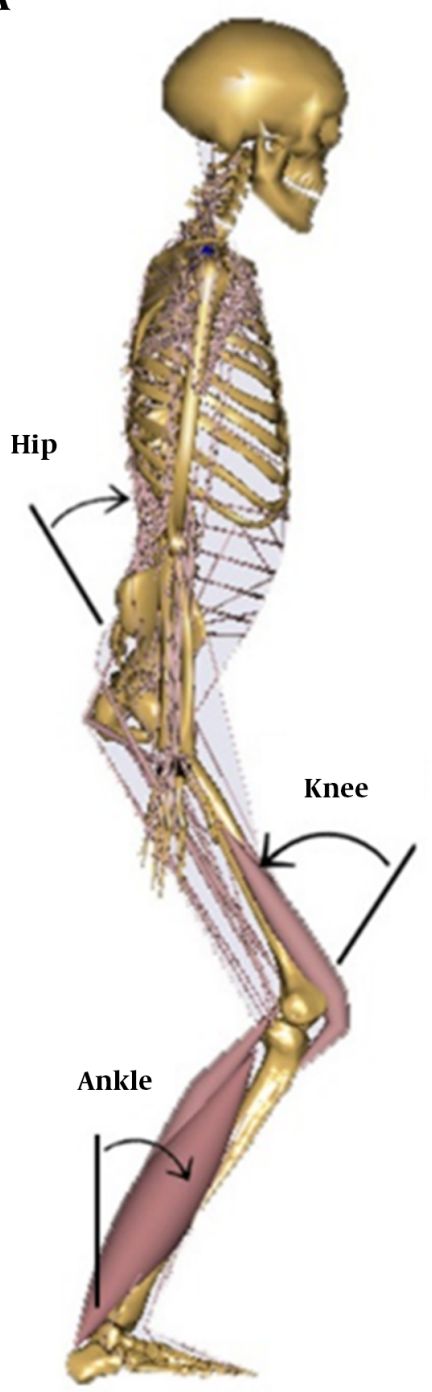

B

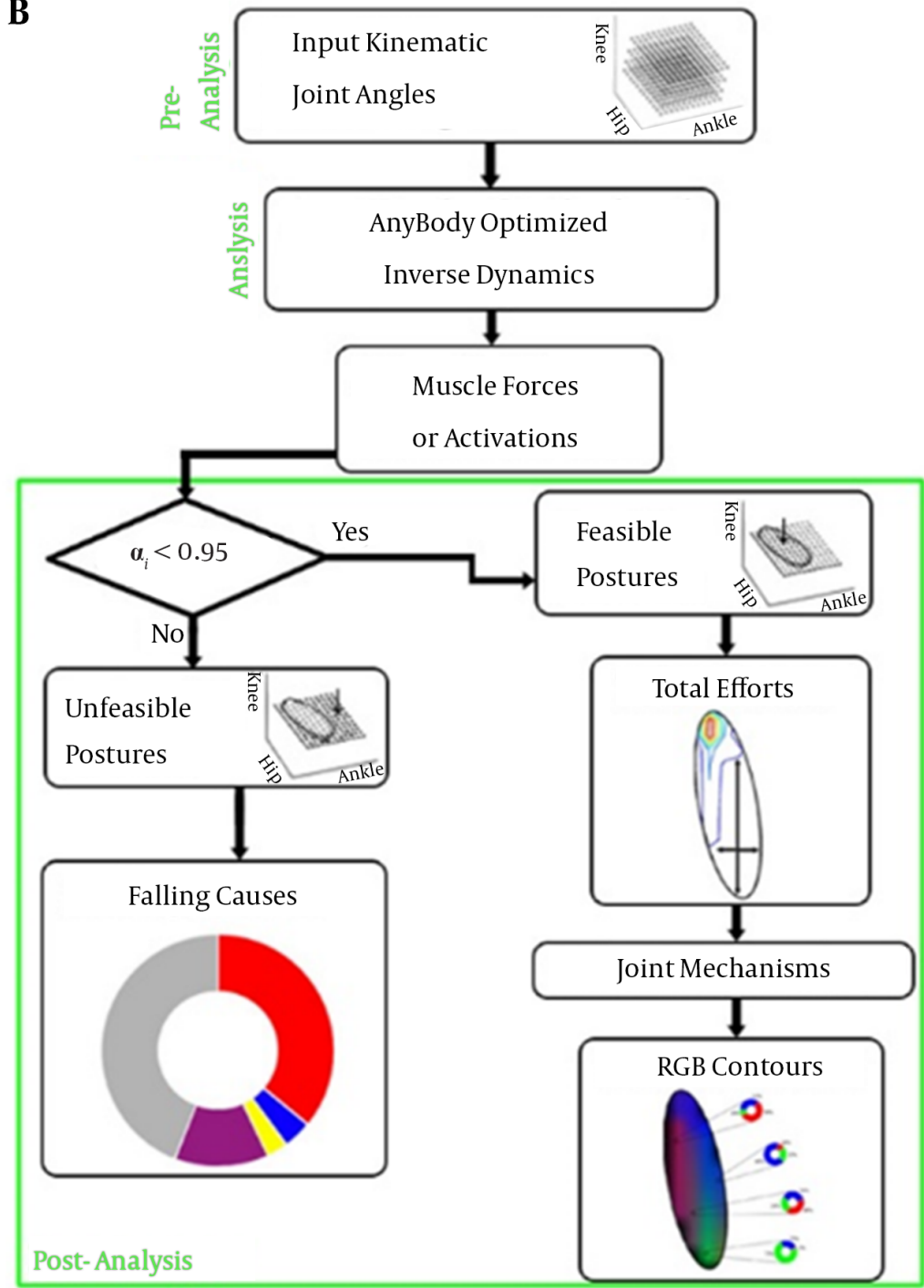

Figure 1. A, Biomechanical model with ISB joint angles; B, Solution flowchart from pre-analysis block for provision of input kinematic data, to analysis block to calculate muscle activations via optimized inverse dynamics, and finally, post-analysis block to filter feasible postures and following representations. The procedure applied for both young and elder models.

\subsection{Joint Mechanisms Contribution}

To determine the role of each mechanism, first, an index of overall effort expenditure was defined among the feasible postures. Sum of squared activations of the muscle acting on the ankle (AE), the knee (KE) and the hip (HE) was considered as the total efforts (TE) needed to provide balanced stance, i.e. Formula 3

$$
T E=A E+K E+H E=\sum_{a n k} \alpha_{i}^{2}+\sum_{k n e} \alpha_{i}^{2}+\sum_{h i p} \alpha_{i}^{2}
$$

Then, contributions of each joint mechanism $\left(\mathrm{C}_{\text {joint }}\right)$ were defined as fractions of the joint-related efforts to the total effort according to Formula 4

$$
C_{a n k}=\frac{A E}{T E}, C_{k n e}=\frac{K E}{T E}, C_{h i p}=\frac{H E}{T E}
$$

\subsection{The RGB Representation}

The joint-related contributions were represented by a graphical mapping from the percentage of the roles to the Red-Green-Blue (RGB) color scale. A home-made code was developed to form each of the three additive matrices of the RGB tensor ranged from 0 - 255 based on the 
relative posture-related contribution of the joint mechanisms. In fact, pure contribution of each joint mechanism was demonstrated by a one of the red, green or blue colors and possible collaboration between them possessed relative blending of these three main colors. Figure $1 \mathrm{~B}$ shows the methodological blocks of the study in brief.

\section{Results}

\subsection{Feasible Postures}

Figure 2A shows the three-dimensional (3D) view of feasible postures in human balance. The young space in considerably greater $(\approx 1.6$ times) than the old. Four cut views from these spaces parallel to ankle-hip planes are shown in Figure 2B. The elders revealed similar behavior to the young after midrange angles of the knee.

\subsection{Total Effort Expenditure}

Standing in each posture required different amount of effort. Figure 3 plots non-uniform distribution of the efforts for young and old models for feasible postures represented by the ellipses. The elders expended more effort, in general, to stand in balanced conditions.

\subsection{Role of Joint Mechanisms}

Relative joint contributions in each feasible ellipse are illustrated in Figure 4. Efforts of the muscles acting on the joints have been mapped into a graphical RGB color scale. Complementary percentages of the roles of each joint mechanism arbitrated related shares of the colors. For instance in the young 24-deg CKA, the knee mechanism (green color) was dominant in lower angles of hip flexion. More knee flexed postures for the young (CKA = $48 \mathrm{deg}$ ) resulted in elimination of the ankle mechanism contributions.

\subsection{Falling Causes}

Analysis on the unfeasible postures could unveil defects of mechanisms in provision of balance in the elders compared to the falls in the young. Figure 5 presents the shares of mechanisms deficiency in stance for two studied age groups. The overall weakness of the muscles acting on the joints was observed in elder's falling causes. Triple interaction of the mechanisms possessed remarkable share of falling causes in the old model.

\section{Discussion}

The present parametric study investigated the physiological effect of aging on the human musculoskeletal system. The aging frailty was modeled by a constant weakening of all muscle based on empirical data in the literature. Although several assumptions and simplifications were taken into account, this study presented a comprehensive framework on the standing and its role-playing mechanisms and also on the falls and their causes.

The main limitation of the study was mere considering the equilibrium condition in the postures and stability was neglected. Therefore, higher levels of co-contraction were not observed in the models. In the assessment of total effort, however, squared activation of all muscles were assumed to involve the effect of antagonistic forces in the static standing postures. Results of this investigation would provide an important step to shed light on the posture-dependent joint contributions in aged model standing studies.

\subsection{Feasible Postures}

The 3D spaces of feasible postures indicated that the old model was more confined to use further angles of the lower joints. The young model was capable to pose in more flexed joints due to its strengthener muscles. In fact, lower amount of physiological muscle tolerance in the elders was led to notably confined maneuverability state-space of the joints. There were several postures specifically with higher ankle and hip angles that the young could provide balance while the old fell.

Cut views of the 3D spaces (Figure 2B) showed that the greater difference between the age groups was in the lower knee angles. In other words, the aged model was more vulnerable to fall when intended to keep the knee straight. By flexing the knee, the elders could own roughly the same feasible region as the young possessed. It thus implied that reducing the height of the body by knee flexion, which was already mentioned within the literature as an involuntary behavior of the old adults in the experiments [29], could be considered as a technique to broaden the standing statespace. But it should be noted that the flexion of the knee may be followed by both reduction in the overall potential energy (seeking a stable situation) and change in the routine muscle recruitment pattern by the CNS due to the changes in the kinematic positions of the joints and muscle fiber lengths.

\subsection{Total Effort Expenditure}

The total effort of the muscles in the aged model was remarkably higher than the young. The old model recruited its muscles at least two-times greater than the 

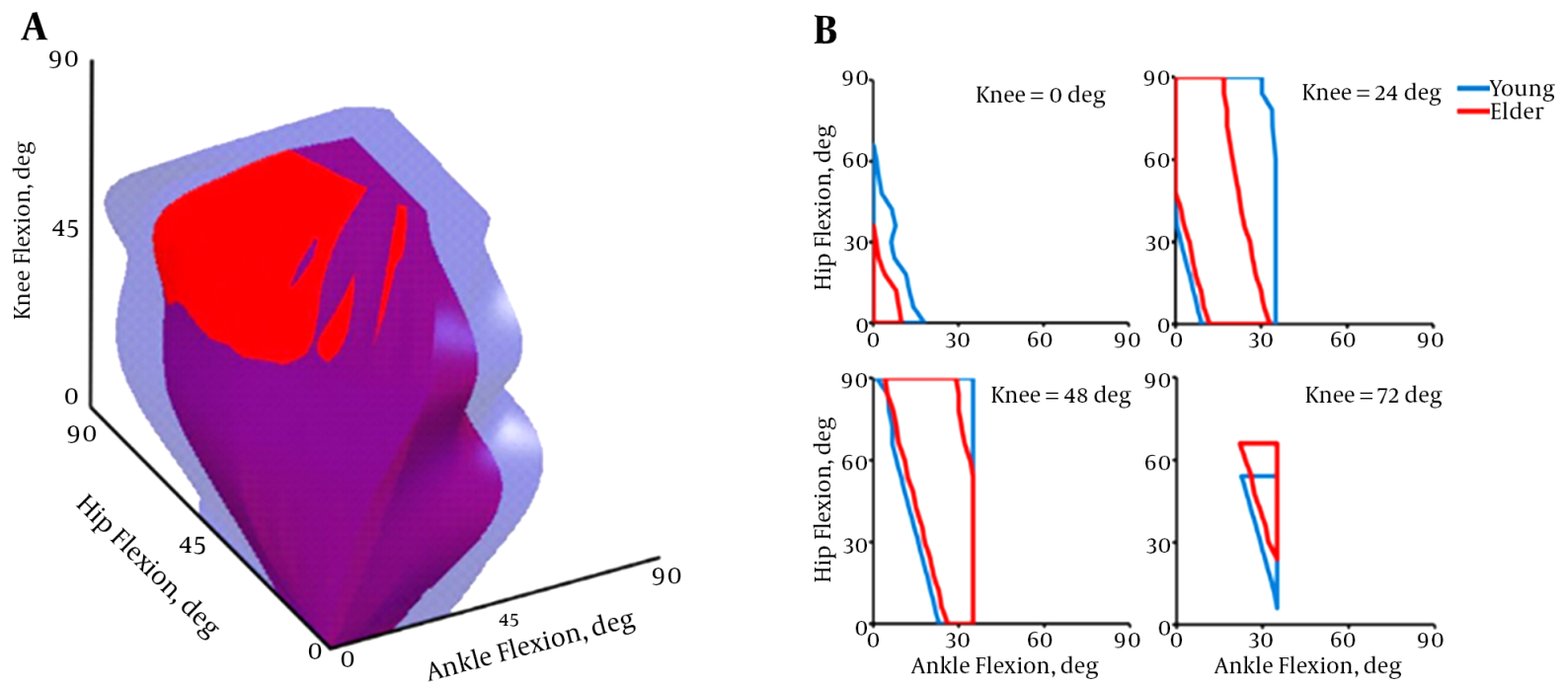

Figure 2. A, 3D feasible space of balanced standing for elder and young models; B, Cut views of feasible regions in four selected CKAs.

young model. It primarily means that an aged model requires more energy expenditure to pose in a same posture in comparison with a healthy young one. McClenaghan et al. (2006) also calculated the power spectral density of the elders in stance and found that the total power was considerably greater in comparison with the young control group [33]. Furthermore, it was shown that the old individuals reveal larger compensatory muscle activation than the young [34]. The lowest 10\% percentile of the efforts (plotted by blue line in Figure 3) indicated the low-cost regions for standing in balance but the old one should expend 2.6 units rather than 1.1 unit for the young. That is, at minimum energy level the elders should consume energy about 2.4 times more. The effect of this consumption may be highlighted in long-term tasks leading to the endurance time of the muscles and fatigue-related consequences.

Dependency of the effort expenditure on the joint angles was more in the elders. For instance, a dramatic decrease (about 20 units) in the effort expenditure was observed by more flexion of the knee from 24 to 48 degrees. In contrast, the young energy consumption was more even through the knee angles. It explicitly declared that flexion of the knee can deeply meliorate the standing condition for the aged people. Such an effect was observed in the young by only changing the posture to slightly reduce the effort. The largest low-cost area of standing in the young model was near 24-degree knee flexion. However, this CKA was shifted to about 48 degrees.

In both age groups, the possibility of changing the posture against the perturbation was highly relied of the hip mechanisms, notably after early CKAs. In 24-degree CKA, the hip joint could be flexed three times more than the ankle in the young. This ratio was encountered by a little increase to 3.5:1 in the old indicating more dependency of the elders to the hip mechanism (see arrows in Figure 3). Such a possibility again was more noticeable in 48-degree knee flexion for the elder model.

\subsection{Roles of Joint Mechanisms}

Collaboration between the joint mechanisms was closely dependent on the standing postures. In the fullextended knee position, the ankle and the hip were dominant in balance provision and the knee was relatively of no contribution due to the absence of the green spectrum colors. The elder standing was a little bit more relied on the hip mechanism in lower ankle and higher hip angles. The previous experimental studies emphasized on use of compensatory mechanisms in elders rather than the mere ankle strategy in the young [35].

In 24-degree flexed knee, the mechanisms possessed their own areas more distinctly than the early CKAs. Vivid and sharp colors in these angles witnessed distinguished roles of the mechanisms besides the blended areas of collaborations. Such a vividness in the colormap was clearer in the young rather than the aged model. Selected postures with quantified contributions of the joints (extracted and illustrated with the RGB doughnut diagrams in Figure $4 \mathrm{~A}$ and $\mathrm{C}$ ) also indicated that the subordinate colors are more contributed to the elder standings unlike the greater dominancy of the main color in the young. In general, the 
A

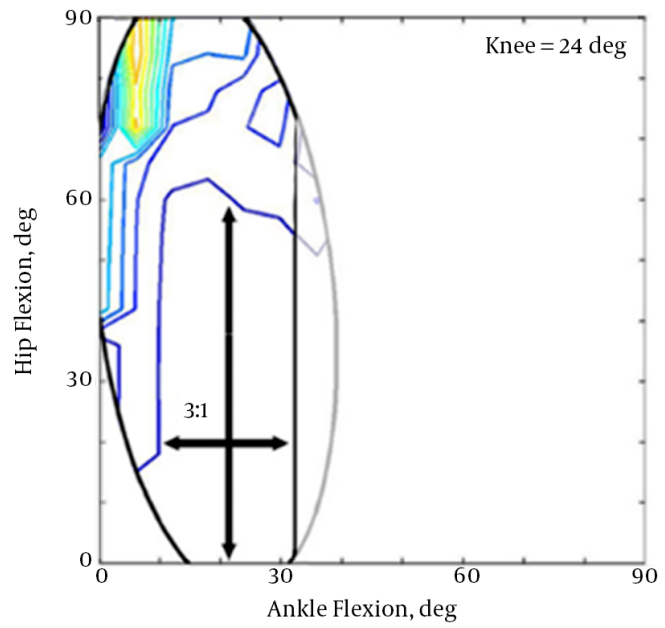

C

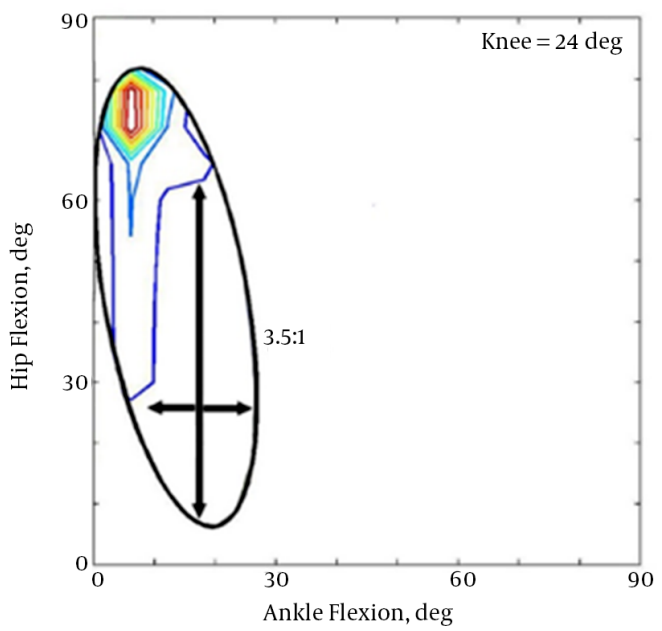

B

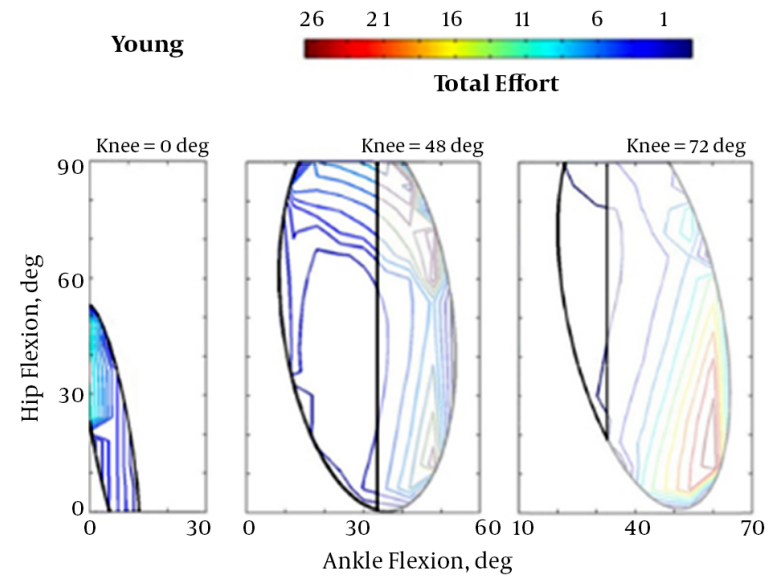

D

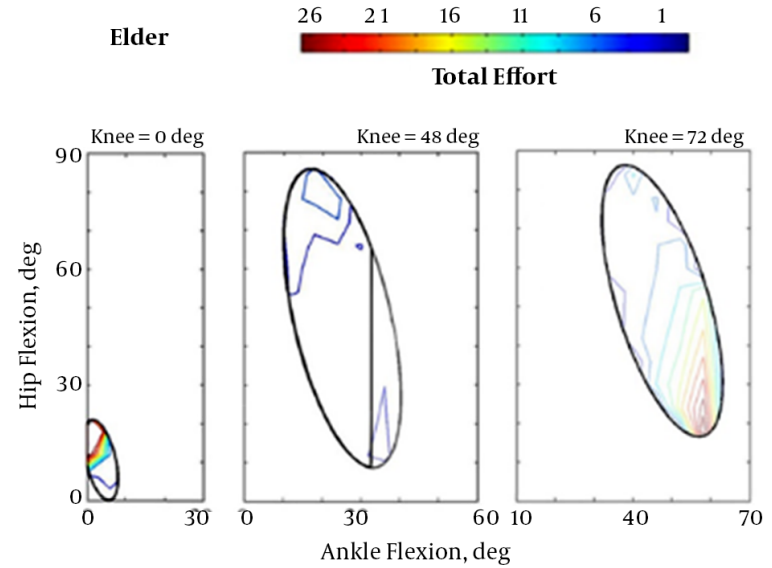

Figure 3. A, Effort distribution for the young model in the $\mathrm{CKA}=24 \mathrm{deg}$; $\mathrm{B}$, Efforts in other young CKAs $=0,48$ and 72 deg; $\mathrm{C}$, Effort distribution for the old model in the CKA $=24 \mathrm{deg}$. $\mathrm{d}$ Efforts in other elder CKAs $=0,48$ and $72 \mathrm{deg}$. The elders' scale bar is two-times greater than the young. Also, crossed arrows within the contours denotes the opportunity of posture change refrained from further effort expenditure.

colormaps of the elders were blurred implying the dependency of the balance on all mechanisms.

After 24-degree knee flexion, the role of the ankle mechanism vanished and collaboration between the knee and the hip became dominant. Higher hip angles were led to role-playing of the hip mechanism, lower angles left for the knee and the mid-range hip angles were the postures that need their collaboration.

\subsection{Falling Causes}

All the falls or unfeasible postures were because of the physiological limitation of the muscles. Interaction of all mechanisms was the overriding reason of the falls (44\%) in the young group. Also, approximately $36 \%$ of falls took place due to pure constrains in the ankle muscle groups like the triceps surae or the tibialis anterior. Although these muscles are intrinsically powerful ones, highly produced moment around the ankle in some postures imposed difficult conditions on these muscles. The body center of mass in more flexed angles has located far from 

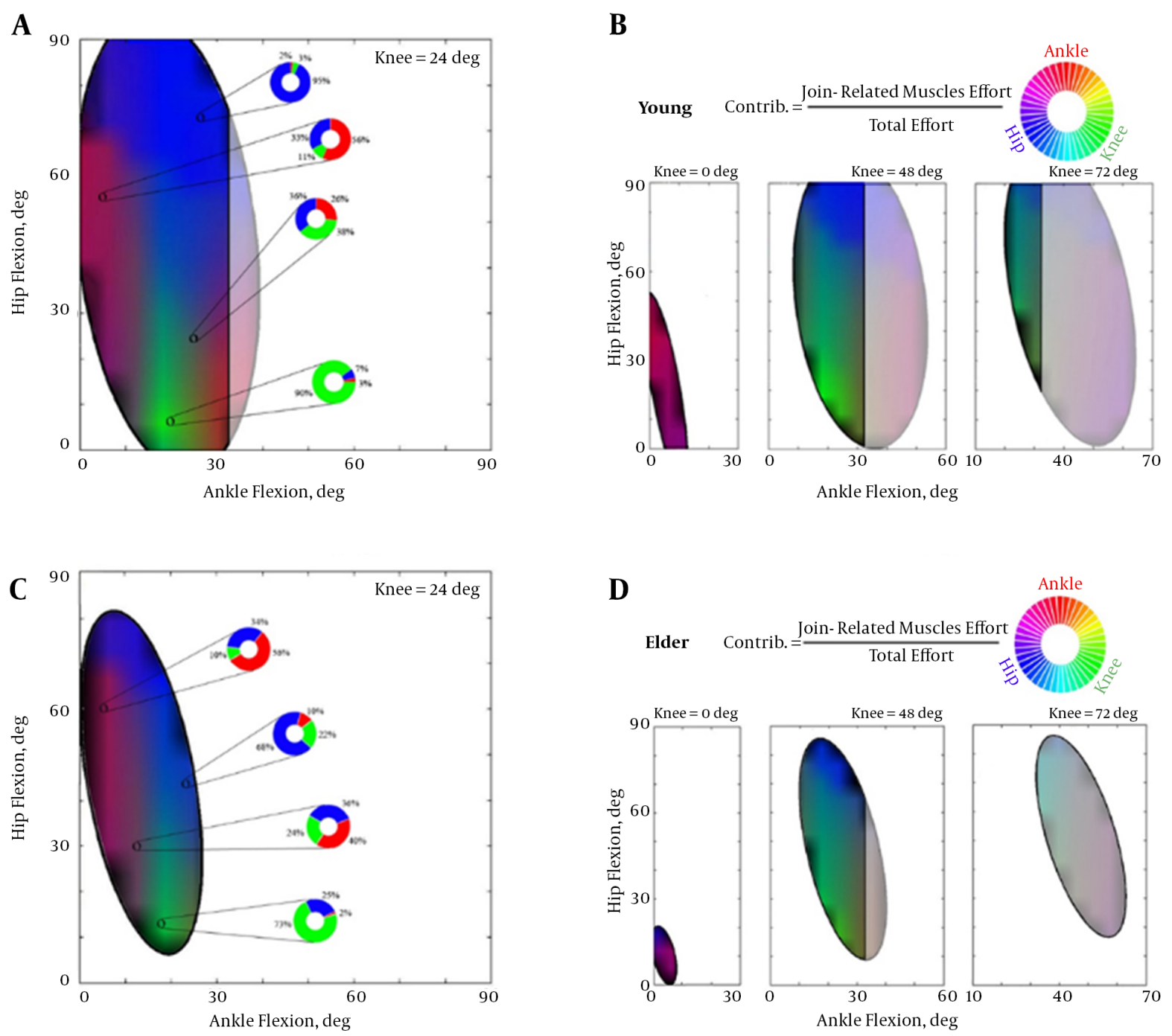

Figure 4. A, RGB representation of joint mechanisms in 24-deg CKA of the young standing; B, And for other CKAs of 0,48 and 72 deg; C Elderly posture-dependent joint mechanisms' role in the RGB colormap for $\mathrm{CKA}=24 \mathrm{deg}$; $\mathrm{D}$, and for $\mathrm{CKAs}=0,48$ and $72 \mathrm{deg}$.

the joint, and hence, provision of equilibrium became impossible. The ankle-hip strategy with $13 \%$ and hip strategy with $4 \%$ of the falling reasons indicated that the knee joint musculature can more properly counteract against the severe conditions imposed by some postures in the balanced standing. The absence of the knee mechanism in the causes of fallen postures confirmed that the knee extensors/flexors are able to withstand against the external moments.

In elders, nevertheless, the triple interaction between the mechanisms was responsible for majority of the falls. It obviously means that the overall weakness of the muscles in the aged model evenly resulted in joint mechanisms. In fact, an age-related fall occurred often due to the simultaneous defects in all mechanisms. The alone contribution of the ankle was the second reason for the age-related falls. Defects in the ankle muscle due to the before-mentioned reasons were similar between the age groups.

In conclusion, contribution of the joint mechanisms was closely relied on the postural position of the body both in the young and the elder models. The aged model had less feasible balanced postures in early knee angles rather than the young but the same behavior observed between them by flexion of the knee. The elders expended more efforts to provide balance and it was deeply dependent upon the knee angle. The optimum knee angle for standing in 


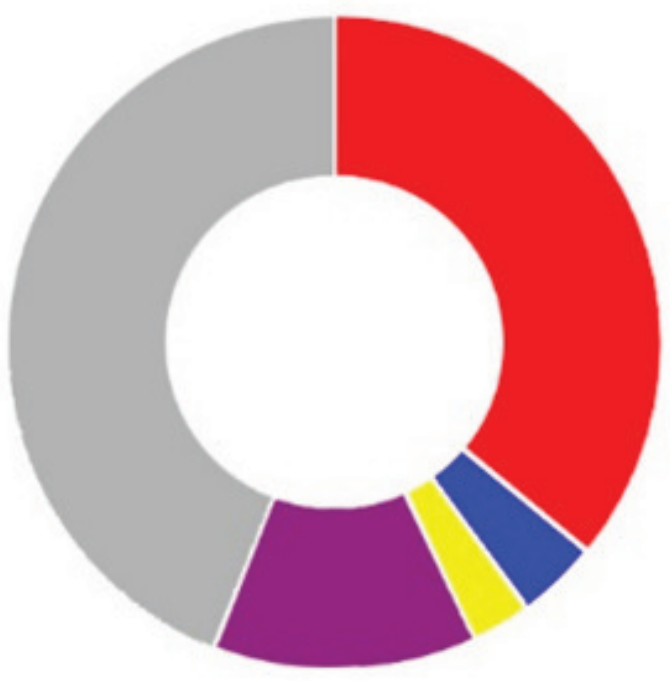

Young

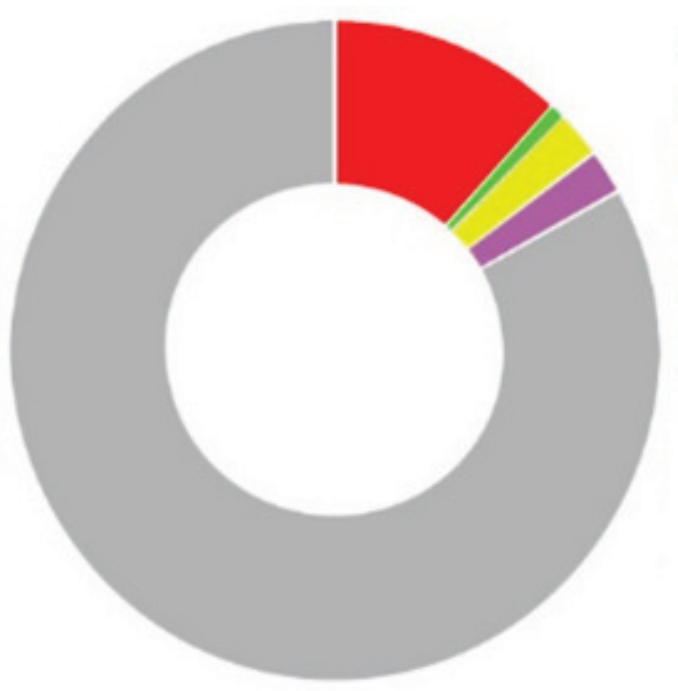

Ankle

Knee

Hip

Ankle*Knee

Ankle*Hip

Knee*Hip

Ankle*Knee*Hip

Elder

Figure 5. Defects in the Balanced Standing for Young and Old Models with Respect to the Joint Mechanisms and Their Interactions

the young model was about 30 degrees while the elders used lower energy in 45-degree knee flexion. The role of the mechanisms was more distinct in the mid-range knee flexion specifically for the young. The elders were imposed to employ more collaboration to master their overall muscle weakness. Age-related falls were closely due to the simultaneous defects in all mechanisms but the ankle was also responsible for the young fallings.

\section{Acknowledgments}

This work was extracted from a PhD dissertation in biomechanics, department of mechanical engineering, Sahand university of technology (code: 90664109) done by MN Ashtiani.

\section{Footnotes}

Authors' Contribution: Dr. Mahmood Reza Azghani developed the study concept, designed, analyzed and interpreted the data. Mr. Mohammed N Ashtiani also performed the modeling, acquired the data, drafted the manuscript and provided technical support. The study is supervised by Dr. Mahmood Reza.

Conflict of Interest: The authors declare no conflict of interest.

\section{Funding/Support: None.}

\section{References}

1. Woollacott MH, Shumway-Cook A, Nashner LM. Aging and posture control: changes in sensory organization and muscular coordination. Int J Aging Hum Dev. 1986;23(2):97-114. doi: 10.2190/VXN3-N3RT54JB-X16X. [PubMed: 3557634].

2. Scott V, Votova K, Scanlan A, Close J. Multifactorial and functional mobility assessment tools for fall risk among older adults in community, home-support, long-term and acute care settings. Age Ageing. 2007;36(2):130-9. doi: 10.1093/ageing/afl165. [PubMed: 17293604].

3. Whipple R, Wolfson L, Derby C, Singh D, Tobin J. Altered sensory function and balance in older persons. J Gerontol. 1993;48 Spec No:71-6. [PubMed: 8409244].

4. Barin K, Dodson EE. Dizziness in the elderly. Otolaryngol Clin North Am. 2011;44(2):437-54. doi:10.1016/j.otc.2011.01.013. [PubMed: 21474016] X.

5. Gilsing MG, Van den Bosch CG, Lee SG, Ashton-Miller JA, Alexander $\mathrm{NB}$, Schultz AB, et al. Association of age with the threshold for detecting ankle inversion and eversion in upright stance. Age Ageing. 1995;24(1):58-66. doi:10.1093/ageing/24.1.58. [PubMed: 7762464].

6. Lexell J, Taylor CC, Sjostrom M. What is the cause of the ageing atrophy? Total number, size and proportion of different fiber types studied in whole vastus lateralis muscle from 15- to 83-year-old men. J Neurol Sci. 1988;84(2-3):275-94. doi: 10.1016/0022-510X(88)90132-3. [PubMed: 3379447].

7. de Rekeneire N, Visser M, Peila R, Nevitt MC, Cauley JA, Tylavsky FA, et al. Is a fall just a fall: correlates of falling in healthy older persons. The Health, Aging and Body Composition Study. J Am Geriatr Soc. 2003;51(6):841-6. doi: 10.1046/j.1365-2389.2003.51267.x. [PubMed: 12757573].

8. McNeil CJ, Doherty TJ, Stashuk DW, Rice CL. Motor unit number estimates in the tibialis anterior muscle of young, old, and very old men. Muscle Nerve. 2005;31(4):461-7. doi: 10.1002/mus.20276. [PubMed: 15685623].

9. Kaya RD, Nakazawa M, Hoffman RL, Clark BC. Interrelationship between muscle strength, motor units, and aging. Exp Gerontol. 2013;48(9):920-5. doi: 10.1016/j.exger.2013.06.008. [PubMed: 23832080]. 
10. Rubenstein LZ, Josephson KR. Risk factors for falls: a central role in prevention. Generations. 2002;26(4):15.

11. Stenholm S, Tiainen K, Rantanen T, Sainio P, Heliovaara M, Impivaara $\mathrm{O}$, et al. Long-term determinants of muscle strength decline: prospective evidence from the 22-year mini-Finland follow-up survey J Am Geriatr Soc. 2012;60(1):77-85. doi: 10.1111/j.1532-5415.2011.03779.x. [PubMed: 22211568].

12. Evans WJ, Lexell J. Human aging, muscle mass, and fiber type composition. J Gerontol A Biol Sci Med Sci. 1995;50 Spec No:11-6. doi: 10.1093/gerona/50A.Special_Issue.11. [PubMed: 7493202].

13. Young A, Stokes M, Crowe M. Size and strength of the quadriceps muscles of old and young women. Eur J Clin Invest. 1984;14(4):282-7. doi: 10.1111/j.1365-2362.1984.tb01182.x. [PubMed: 6434323].

14. Young A, Stokes M, Crowe M. The size and strength of the quadriceps muscles of old and young men. Clin Physiol. 1985;5(2):145-54. doi: 10.1111/j.1475-097X.1985.tb00590.x. [PubMed: 3888498].

15. Frontera WR, Hughes VA, Lutz KJ, Evans WJ. A cross-sectional study o muscle strength and mass in 45- to 78-yr-old men and women. J Appl Physiol (1985). 1991;71(2):644-50. [PubMed: 1938738].

16. Horlings CG, van Engelen BG, Allum JH, Bloem BR. A weak balance: the contribution of muscle weakness to postural instability and falls. Nat Clin Pract Neurol. 2008;4(9):504-15. doi: 10.1038/ncpneuro0886. [PubMed: 18711425].

17. Regterschot GR, Folkersma M, Zhang W, Baldus H, Stevens M, Zijlstra W. Sensitivity of sensor-based sit-to-stand peak power to the effects of training leg strength, leg power and balance in older adults. Gait Posture. 2014;39(1):303-7. doi: 10.1016/j.gaitpost.2013.07.122. [PubMed: 23973356].

18. Wolfson L, Judge J, Whipple R, King M. Strength is a major factor in balance, gait, and the occurrence of falls. J Gerontol A Biol Sci Med Sci. 1995;50 Spec No:64-7. doi: 10.1093/gerona/50A.Special_Issue.64. [PubMed: 7493221].

19. Onambele GL, Narici MV, Maganaris CN. Calf muscle-tendon properties and postural balance in old age. J Appl Physiol (1985). 2006;100(6):2048-56. doi: 10.1152/japplphysiol.01442.2005. [PubMed: 16455811].

20. Horak FB, Shupert CL, Mirka A. Components of postural dyscontrol in the elderly: a review. Neurobiol Aging. 1989;10(6):727-38. doi 10.1016/0197-4580(89)90010-9. [PubMed: 2697808].

21. Manchester D, Woollacott M, Zederbauer-Hylton N, Marin O. Visual, vestibular and somatosensory contributions to balance control in the older adult. J Gerontol. 1989;44(4):M118-27. [PubMed: 2786896].

22. Amiridis IG, Hatzitaki V, Arabatzi F. Age-induced modifications of static postural control in humans. Neurosci Lett. 2003;350(3):137-40. doi: 10.1016/S0304-3940(03)00878-4. [PubMed: 14550913].

23. Woollacott MH, Shumway-Cook A. Changes in posture control across the life span-a systems approach. Phys Ther. 1990;70(12):799-807. doi: 10.1093/ptj/70.12.799. [PubMed: 2236223].

24. Lin SI, Woollacott MH, Jensen JL. Postural response in older adults with different levels of functional balance capacity. Aging Clin Exp Res. 2004;16(5):369-74. doi: 10.1007/BF03324566. [PubMed: 15636462].

25. Davidson PM, Digiacomo M, McGrath SJ. The feminization of aging: how will this impact on health outcomes and services? Health Care Women Int. 2011;32(12):1031-45. doi: 10.1080/07399332.2011.610539. [PubMed: 22087593].

26. Qu X, Nussbaum MA. Effects of external loads on balance control during upright stance: experimental results and model-based predictions. Gait Posture. 2009;29(1):23-30. doi: 10.1016/j.gaitpost.2008.05.014. [PubMed: 18632273].

27. Cenciarini M, Loughlin PJ, Sparto PJ, Redfern MS. Stiffness and damping in postural control increase with age. IEEE Trans Biomed Eng. 2010;57(2):267-75. doi: 10.1109/TBME.2009.2031874. [PubMed: 19770083].

28. Iqbal K, Pai Y. Predicted region of stability for balance recovery: motion at the knee joint can improve termination of forward movement. J Biomech. 2000;33(12):1619-27. doi: 10.1016/S0021-9290(00)00129-9. [PubMed: 11006386].

29. Di Giulio I, Baltzopoulos V, Maganaris CN, Loram ID. Human standing: does the control strategy preprogram a rigid knee? J Appl Physiol (1985). 2013;114(12):1717-29. doi: 10.1152/japplphysiol.01299.2012. [PubMed: 23620493].

30. Wu G, Siegler S, Allard P, Kirtley C, Leardini A, Rosenbaum D, et al. ISB recommendation on definitions of joint coordinate system of various joints for the reporting of human joint motionpart I: ankle, hip, and spine. International Society of Biomechanics. J Biomech. 2002;35(4):543-8. doi: 10.1016/S0021-9290(01)00222-6. [PubMed: 11934426].

31. Damsgaard M, Rasmussen J, Christensen ST, Surma E, de Zee M. Analysis of musculoskeletal systems in the anybody modeling system. Simul Model Pract Theor. 2006;14(8):1100-11. doi: 10.1016/j.simpat.2006.09.001.

32. Devita P, Skelly WA. Effect of landing stiffness on joint kinetics and energetics in the lower extremity. Med Sci Sports Exerc. 1992;24(1):10815. doi:10.1249/00005768-199201000-00018. [PubMed:1548984].

33. McClenaghan BA, Williams HG, Dickerson J, Dowda M, Thombs L, Eleazer P. Spectral characteristics of aging postural control. Gait Posture. 1996;4(2):112-21. doi: 10.1016/0966-6362(95)01040-8.

34. Kanekar N, Aruin AS. The effect of aging on anticipatory postural control. Exp Brain Res. 2014;232(4):1127-36. doi: 10.1007/s00221-014-3822-3. [PubMed: 24449006]

35. Blaszczyk JW, Michalski A. Aging and postural stability. Stud Phys Cult Tourism. 2006;13:11-4. 